\title{
Fermi-Surface Mapping from 2D Positron Annihilation Data
}

\author{
G. Kontrym-Sznajd and M. Samsel-CzeKąA
}

W. Trzebiatowski Institute of Low Temperature and Structure Research Polish Academy of Sciences, P.O. Box 1410, 50-950 Wrocław 2, Poland

We demonstrate which kind of information on the electronic structure one can get from one-dimensional profiles, interpreted in terms of two-dimensional reconstructed densities. The conversion from one-dimensional to two-dimensional is applied to one-dimensional angular correlation of annihilation radiation profiles of divalent hexagonal close packed metals $\mathrm{Mg}$ and $\mathrm{Cd}$. On the example of $\mathrm{Mg}$ we show that one should be very careful while studying the Fermi surface from electron-positron (e-p) densities folded into the first Brillouin zone.

PACS numbers: 71.18.+y, 13.60.Fz, 87.59.Fm

\section{Introduction}

One-dimensional (1D) spectra, representing plane integrals of 3D electron momentum densities $\rho(\boldsymbol{p})$, are measured either in the Compton scattering or 1D angular correlation of annihilation radiation (ACAR) experiment. In principle the reconstruction of 3D densities from their plane projections [1-6] demands a large number of profiles. Thus, for $1 \mathrm{D}$ data, we recommended also the reconstruction of $2 \mathrm{D}$ density $[7,8]$, defined as

$$
\rho^{\boldsymbol{L}}\left(p_{z}, p_{y}\right) \equiv J\left(p_{z}, p_{y}\right)=\int_{-\infty}^{\infty} \rho(\boldsymbol{p}) \mathrm{d} p_{x}
$$

where 1D spectrum, being a plane integral of 3D density $\rho(\boldsymbol{p})$, is treated as a line integral of the $2 \mathrm{D}$ density $\rho^{\boldsymbol{L}}$

$$
J\left(p_{z}\right)=\int_{-\infty}^{\infty} \int_{-\infty}^{\infty} \rho(\boldsymbol{p}) \mathrm{d} p_{x} \mathrm{~d} p_{y}=\int_{-\infty}^{\infty} \rho^{\boldsymbol{L}}\left(p_{z}, p_{y}\right) \mathrm{d} p_{y} .
$$

To reproduce $\rho^{\boldsymbol{L}}$ efficiently from the smallest number of projections (the same mathematical problem as in transverse tomography), one should measure $J\left(p_{z}\right)$ 
for $p_{z}$ perpendicular to the main rotation axis ([001] direction). It allows us to reconstruct $\rho^{L=[001]} \equiv \rho^{001}$ from a small number of profiles. It has been checked for yttrium profiles (even three 1D ACAR profiles were quite sufficient to reproduce properly $\rho^{001}[7]$ ) and next applied to Compton profiles in $\mathrm{Cr}[8]$ and Be [9] while using the Cormack reconstruction algorithm [10]. In the Cormack method data are expanded into orthogonal polynomials - the uniqueness of this expansion (with some practical formulae) is described in the Appendix.

Finally, to obtain momentum densities in the reduced space $\boldsymbol{k}$, the LockCrisp-West (LCW) transformation was performed [11], i.e. a conversion from the extended $\boldsymbol{p}$ to reduced $\boldsymbol{k}$ space. In the case of electron density $\rho^{\boldsymbol{L}}(\boldsymbol{k})$ represents the sum (over occupied bands) of line dimensions of the electron Fermi surface (FS) along $\boldsymbol{L}$. However, in the case of e-p densities line dimensions of the electron FS in the band $j$ th must be modified by some function $f_{j}(\boldsymbol{k})$ which contains both many-body and positron wave function effects. We will demonstrate that when $f_{j}(\boldsymbol{k})$ is strongly momentum dependent (as e.g. in $\mathrm{Mg}$ ), one should be very careful drawing the FS from $\rho^{\boldsymbol{L}}(\boldsymbol{k})$.

\section{Results}

1D ACAR spectra for magnesium and cadmium were created from 2D ACAR data [12] measured for $p_{z}$ along [100] and [110] directions and with full width at half of maximum (FWHM) of 0.1 in atomic units of momentum (a.u.). From two 1D profiles $J\left(p_{z}\right)$ (with $p_{z}$ along [100] and [110]) 2D e-p momentum densities $\rho^{001}(\boldsymbol{p})$ were reconstructed and LCW densities $\rho^{001}(\boldsymbol{k})$, presented in Fig. 1 for the models and the real data, were created.

The free-electron FS of divalent hcp metals with the axial ratio $r=c / a$ lower than $r_{\mathrm{c}}=(3.375 \sqrt{3}) / \pi=1.860735$ contains the following elements [13]: 1st zone holes around $\mathrm{H}$ points (caps); 2nd zone hole (monster); 3rd zone electrons around: $\Gamma$ (lens), $L$ (butterflies), $K$ (needles) and 4 th zone electron pockets around $L$. In the case of Mg the free-electron FS contains all these elements while for Cd the needles do not exist due to $r$ higher than the critical value $r_{\mathrm{c}}$ at which they disappear. However, because these needles (in $\mathrm{Mg}$, Be, and $\mathrm{Zn}$ ) are small, free-electron densities $\rho^{001}(\boldsymbol{k})$ in all divalent hcp metals are very similar (as displayed in Fig. 1a). There is no such similarity for the "real" densities - compare in Fig. 1 parts (b) and (c) with (a).

According to the band structure results [14], in Cd there is a lack of butterflies and pockets when compared to the free-electron model, clearly seen also from reconstructed densities $\rho_{\mathrm{e}-\mathrm{p}}^{001}(\boldsymbol{k})$ (if they exist, densities along $M L$ line should be higher than along $\Gamma A-$ compare Fig. $1 \mathrm{c}$ and $\mathrm{c}^{\prime}$ ). Meanwhile, in the case of $\mathrm{Mg}$, where the results should be very similar to the free electron ones [14, 15], $2 \mathrm{D}$ densities $\rho^{001}(\boldsymbol{k})$ are very surprising (Fig. 1b). Namely, it seems that $\rho_{\mathrm{e}-\mathrm{p}}^{001}(\boldsymbol{k})$, reconstructed from two 1D ACAR spectra, either do not contain the lens around $\Gamma$ 


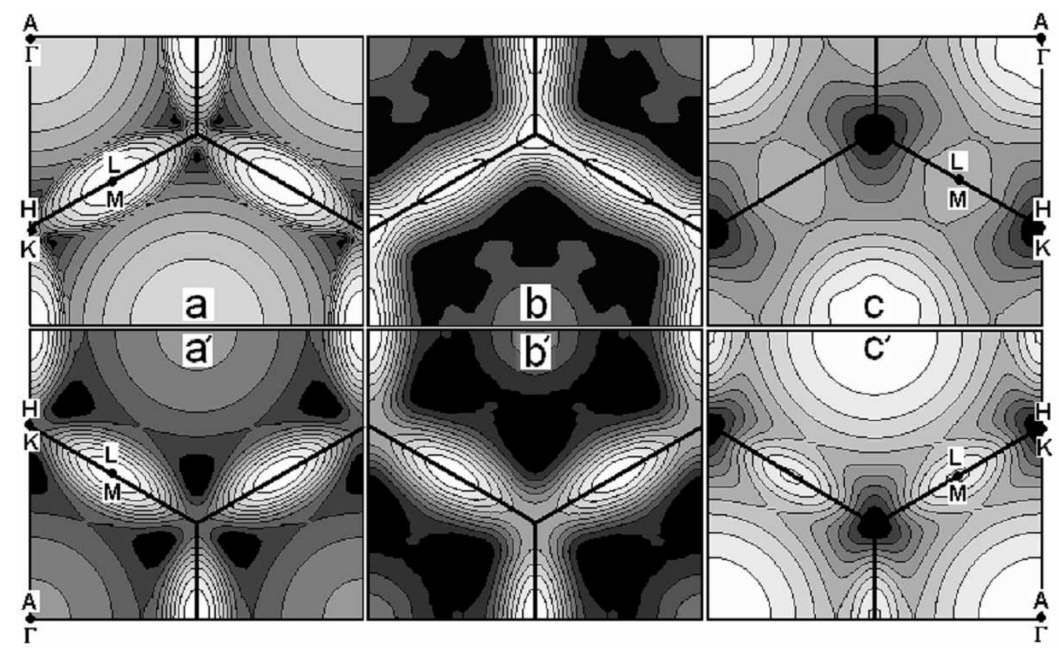

Fig. 1. $\rho^{001}(\boldsymbol{k})$ in $\mathrm{Mg}(\mathrm{a}, \mathrm{b})$ and $\mathrm{Cd}(\mathrm{c})$ for models and real $\mathrm{e}-\mathrm{p}$ reconstructed densities. For Mg: (a) - free electron model; $\left(\mathrm{a}^{\prime}\right)$ - model (a) with a Kahana-like enhancement and convoluted with the experimental FWHM = 0.1 a.u.; (b) - reconstructed from two 1D ACAR profiles; ( $\left.\mathrm{b}^{\prime}\right)$ - isotropic part of the densities (b). For Cd: (c) densities reconstructed from two 1D ACAR spectra; $\left(c^{\prime}\right)$ - free-electron model (without enhancement) after convoluting. All isodensities are drawn by 11 contour lines.

or it is much reduced in comparison with the free-electron model. Our tests showed that it is connected with a strongly momentum dependent $\mathrm{e}-\mathrm{p}$ enhancement factor as well as smearing effects (resolution broadening and, the most likely, electronelectron (e-e) correlations) drawn in Fig. 1a'. The e-e correlations, similar to the Daniel and Vosko effect [16], were observed for $\mathrm{Mg}$ also in Compton profiles [17] (the Lam-Platzman correction [18] partly improved agreement between theory and experiment). These effects that, of course, do not change the FS itself but only densities, minimize a relative contribution of lens around the $\Gamma$ point. As a result, model densities in Fig. 1 without (a) and with both Kahana-like e-p enhancement and smearing $\left(\mathrm{a}^{\prime}\right)$ differ essentially from each other and $\left(\mathrm{a}^{\prime}\right)$ becomes more similar to the reconstructed densities $\left(b^{\prime}\right)$. Thus, particularly in the case of ACAR data, which contain also "positron effects" (positron wave-function and e-p correlation effects), we should be very careful drawing the FS from either 1D or 2D densities, even though in the case of 3D densities there is an enough high jump of $\rho(\boldsymbol{k})$ on the FS [19] (in our case this jump was even enhanced by the e-p correlations).

\section{Conclusions}

We point out that sometimes interpretation of $1 \mathrm{D}$ or $2 \mathrm{D}$ ACAR data (in terms of FS derived from LCW folded densities) can make some difficulties, demonstrated here on the example of $\mathrm{Mg}$ and observed also for $\mathrm{Cr}$ [8]. Dugdale et al. [8] 
got for $\mathrm{Cr}$ a discrepancy between $2 \mathrm{D}$ electron $\left(\rho^{011}(\boldsymbol{k})\right.$ reconstructed from seven $1 \mathrm{D}$ Compton profiles) and e-p densities (2D ACAR data folded into the 1st Brillouin zone). Because in both cases (Compton and positron annihilation) smearing effects (resolution broadening and e-e correlations) should be the same [20], one could expect this being connected either with e-p correlations or positron wave function effects. However, since in $\mathrm{Cr}$ the effective enhancement factor is not momentum dependent [20, 21], it seems that the observed effect [8] follows from either a positron wave function (this can be checked by simple calculations within the independent particle model) or selectivity of the enhancement — in both cases selectivity of positron annihilation with $d$ - and $s$-like states.

\section{Appendix}

The uniqueness of expanding some function into Chebyshev polynomials series is connected with the fact that they are the only polynomials which zeros are known analytically. So, the coefficients of the expansion can be calculated not only with a high accuracy (applying analytically the Gauss quadratures of the arbitrarily high order) but also in a very simple way. Moreover, these are orthogonal polynomials, so they have mean-squares approximation properties it is very important for real data which contain experimental noise.

In Cormack's method data are expanded into a series of even Chebyshev polynomials of the 2 nd kind, $U_{2 n}(p)$, multiplied by their weight:

$$
f(p)=\sum_{n=0}^{\infty} a_{n} \sqrt{1-p^{2}} U_{2 n}(p) .
$$

The coefficient $a_{n}$ can be evaluated from the orthogonality relation, i.e.

$$
a_{n}=\frac{2}{\pi} \int_{0}^{1} f(p) U_{2 n}(p) \mathrm{d} p
$$

In the unit system $[0,1]$, i.e. for $p \in\left[0, p_{z} / p_{z}^{\max }\right]$ where $p_{z}^{\max }$ denotes a momentum for which $f\left(p_{z}\right)$ is close to zero, one can introduce a new unit system where $p=\cos (\alpha)$ with $\alpha$ changing from $90^{\circ}$ to $0^{\circ}$. Now Eqs. (A1) and (A2) become:

$$
\begin{aligned}
& f(p \equiv \cos (\alpha))=\sum_{n=0}^{\infty} a_{n} \sin ((2 n+1) \alpha), \\
& a_{n}=\frac{2}{\pi} \int_{0}^{1} f(\cos (\alpha)) \sin ((2 n+1) \alpha) \mathrm{d} \alpha
\end{aligned}
$$

and instead of calculating the integral (A2) with a function $U_{2 n}(p)$ which zeros are not equally spaced, we have the integral (A4) with a function sin whose zeros are equidistant. Such an integral can be simply replaced by the sum:

$$
a_{n}=\frac{2}{M}\left[\sum_{i=1}^{M-1} f\left(\cos \left(\mathrm{i} \Delta_{\alpha}\right)\right) \sin \left((2 n+1) \mathrm{i} \Delta_{\alpha}\right)+\frac{1}{2}(-1)^{M} f(0)\right]
$$


with a constant value of $\Delta_{\alpha}$ where (for $\Delta_{\alpha}$ in degree) $M=90^{\circ} / \Delta_{\alpha}$. In this case also derivatives (with respect to $p$ ) can be evaluated in a simple way:

$$
\begin{aligned}
& f^{\prime}(p)=-\frac{1}{\sin \alpha} \sum_{n=0}^{\infty} a_{n} m \cos (m \alpha) \text { and }-\frac{f^{\prime}(p)}{p}=\frac{2}{\sin 2 \alpha} \sum_{n=0}^{\infty} a_{n} m \cos (m \alpha), \\
& f^{\prime \prime}(p)=-\frac{1}{\sin ^{3} \alpha} \sum_{n=0}^{\infty} a_{n} m[\cos (m \alpha) \cos \alpha+m \sin \alpha \sin (m \alpha)] \\
& f^{\prime \prime \prime}(p)=\frac{1}{\sin ^{3} \alpha} \sum_{n=0}^{\infty} a_{n} m\left[\cos (m \alpha)\left(m^{2}-1-3 \frac{\cos ^{2} \alpha}{\sin ^{2} \alpha}\right)-3 m \frac{\cos \alpha}{\sin \alpha} \sin (m \alpha)\right]
\end{aligned}
$$

with $m=2 n+1$. All these quantities are not determined only for $p$ close to the unity $\left(p_{z}\right.$ close to $p_{z}^{\max }$ ). However, for $p \approx 1$ they must be equal to zero.

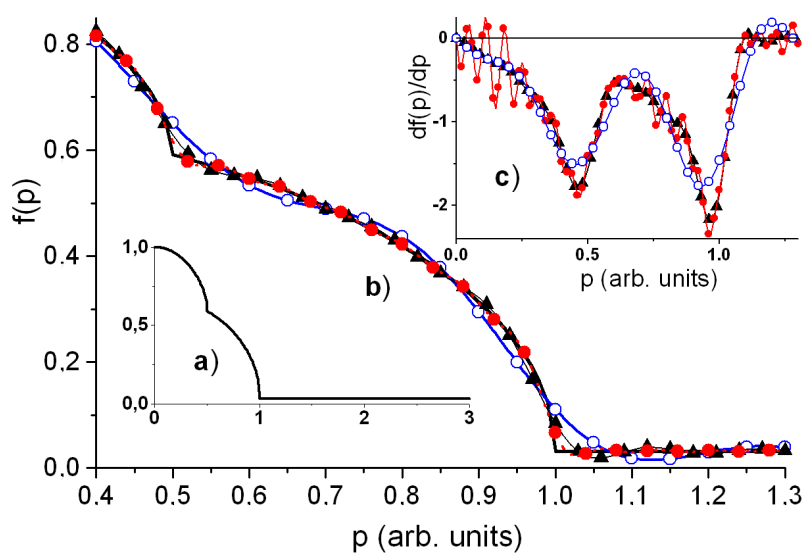

Fig. 2. Model function (part (a) and solid line in (b)) and its 1st derivative (part (c)), described by 30, 60, and 150 polynomials $U_{2 n}(p)$ - open circles, solid triangles, and solid circles, respectively. In the case (c) the model function contains simulated noise and is smeared by FWHM $=0.1$ (the smeared function is perfectly described by 60 polynomials).

Derivatives (see Fig. 2) could help us to observe the similarity between theory and experiment (all details of $J\left(p_{z}\right)$ are bulged by its derivatives) as well as to estimate the most efficient number of the expansion coefficients, particularly for experimental data which always contain statistical noise. The 1st derivative of some model function, calculated in the way proposed in the paper is presented in Fig. 2c. It shows that in our case (the model with simulated statistical noise) it is reasonable to use 60 polynomials to both describe the model function and to "remove" the noise. 


\section{References}

[1] P.E. Mijnarends, Phys. Rev. 160, 512 (1967).

[2] N.K. Hansen, P. Pattison, J.R. Schneider, Z. Phys. B, Condens. Matter 66, 305 (1987).

[3] Y. Tanaka, N. Sakai, Y. Kubo, H. Kawata, Phys. Rev. Lett. 70, 1537 (1993).

[4] L. Dobrzyński, A. Holas, Nucl. Instrum. Methods Phys. Res. A 383, 589 (1996).

[5] G. Reiter, R. Silver, Phys. Rev. Lett. 54, 1047 (1985).

[6] G. Kontrym-Sznajd, M. Samsel-Czekała, Appl. Phys. A 70, 89 (2000).

[7] G. Kontrym-Sznajd, R.N. West, S.B. Dugdale, Mater. Sci. Forum 255-257, 796 (1997); G.Kontrym-Sznajd, in: First International Workshop on High Resolution Compton Scattering as a Probe of Fermiology, Kraków (Poland) 1993 (unpublished).

[8] S.B. Dugdale, H.M. Fretwell, K.J. Chen, Y. Tanaka, A. Shukla, T. Buslaps, Ch. Bellin, G. Loupias, M.A. Alam, A.A. Manuel, P. Suortti, N. Shiotani, J. Phys. Chem. Solids 61, 361 (2000).

[9] G. Kontrym-Sznajd, M. Samsel-Czekała, S. Huotari, K. Hämäläinen, S. Manninen, Phys. Rev. B 68, 155106 (2003) and references therein.

[10] A.M. Cormack, J. Appl. Phys. 34, 2722 (1963); 35, 2908 (1964); G. Kontrym-Sznajd, Phys. Status Solidi A 117, 227 (1990).

[11] D.G. Lock, V.H.C. Crisp, R.N. West, J. Phys. F, Met. Phys. 3, 561 (1973).

[12] P.A. Walters, J. Mayers, R.N. West, in: Positron Annihilation, Eds. P.G. Coleman, S.C. Sharma, L.M. Diana, North-Holland, Amsterdam 1982, p. 334.

[13] W.A. Harrison, Phys. Rev. 126, 497 (1962).

[14] S. Daniuk, T. Jarlborg, G. Kontrym-Sznajd, J. Majsnerowski, H. Stachowiak, J. Phys. Condens. Matter 1, 8397 (1989) and references therein.

[15] I. Baraille, C. Pouchan, M. Causa, F. Marrinelli, J. Phys., Condens. Matter 10, 1096 (1998).

[16] E. Daniel, S.H. Vosko, Phys. Rev. 120, 2041 (1960).

[17] M. Matsumoto, T. Sano, S. Wakoh, J. Phys. Soc. Jpn. 68, 1035 (1999).

[18] L. Lam, P.M. Platzman, Phys. Rev. B 9, 5122 (1974).

[19] L.P.L.M. Rabou, P.E. Mijnarends, Solid State Commun. 52, 933 (1984); J.H. Kaiser, R.N. West, N. Shiotani, J. Phys. F, Met. Phys. 16, 1307 (1986).

[20] H. Sormann, G. Kontrym-Sznajd, R.N. West, Mater. Sci. Forum 363-365, 609 (2001); G. Kontrym-Sznajd, H. Sormann, Acta Phys. Pol. A 107, (2005).

[21] A. Rubaszek, Z. Szotek, W.M. Temmerman, Phys. Rev. B 65, 125104 (2002). 